\title{
Effect of micro-crack and reaction product on laser damage performance of optical glass during chemical etching
}

Huapan Xiao, Zhi Chen, Hairong Wang, Rongguang Liang, $\mathrm{Na} \mathrm{Yu}$, et al.

Huapan Xiao, Zhi Chen, Hairong Wang, Rongguang Liang, $\mathrm{Na}$ Yu, Jiuhong Wang, "Effect of micro-crack and reaction product on laser damage performance of optical glass during chemical etching," Proc. SPIE 10713, Pacific-Rim Laser Damage 2018: Optical Materials for High-Power Lasers, 1071309 (16 May 2018); doi: 10.1117/12.2316622

SPIE Event: Pacific Rim Laser Damage 2018: Optical Materials for High Power Lasers, 2018, Yokohama, Japan 


\title{
Effect of micro-crack and reaction product on laser damage performance of optical glass during chemical etching
}

\author{
Huapan Xiao ${ }^{\mathrm{a}, \mathrm{b}}$, Zhi Chen ${ }^{\mathrm{a}, \mathrm{b}}$, Hairong Wang ${ }^{\text {a,b }}$, Rongguang Liang ${ }^{\mathrm{c}}$, Na Yu $\mathrm{u}^{\mathrm{a}, \mathrm{b}}$, Jiuhong Wang, \\ ${ }^{a}$ State Key Laboratory for Manufacturing Systems Engineering, Xi'an Jiaotong University, Xi'an, \\ Shaanxi 710049, China; 'School of Mechanical Engineering, Xi'an Jiaotong University, Xi'an, \\ Shaanxi 710049, China; ${ }^{\mathrm{c} C o l l e g e}$ of Optical Sciences, University of Arizona, Tucson, Arizona \\ 85721, USA \\ *whairong@xjtu.edu.cn
}

\begin{abstract}
Chemical etching is usually utilized to improve the laser damage performance of optical glass by mitigating microcracks, while it inevitably produces some reaction products (RPs). In this paper, two K9 glasses with good quality and two K9 glasses with micro-cracks are etched, statically or dynamically (high-frequency ultrasonic agitation). The morphologies of cracks and RPs are characterized, and the laser-induced damage thresholds (LIDTs) are measured. The results show that with the increase of etching time, the LIDT increases slightly at first and then decreases gradually for the glass with RPs, and the LIDT increases at first and then stabilizes for the glass without RPs. Using finite-difference time-domain method, the light intensities around crack, RP and their combination are simulated, respectively. The results indicate that the light intensity enhancement factor (LIEF) increases at first and then decreases with the decrease of crack aspect ratio, and the LIEF increases with RP radius. The LIEF for the combination is generally larger than that for one crack or one RP, which greatly depends on the relative distance between the crack and the RP. Experimental and simulated results complement each other, revealing the influence mechanism of crack and RP on the LIDT. This work would contribute to improving the LIDT of optical glass by chemical etching.
\end{abstract}

Keywords: optical glass, chemical etching, laser damage, light intensity, micro-crack

\section{INTRODUCTION}

Some defects are usually generated below the surface of optical glass during their grinding and polishing processes. These defects are referred to as subsurface damage (SSD), which is dominated by subsurface micro-cracks ${ }^{1-2}$. In the field of high-power laser applications, subsurface micro-cracks can greatly reduce the laser damage performance of optical glass, which is usually used as transmitting lens or debris shield ${ }^{3-4}$. Therefore, how to manufacture the optical glass with few SSDs becomes a research hotspot for many research institutions.

Chemical etching is usually regarded as an effective process to reduce or eliminate SSDs in optical glass by opening, blunting and coalescing micro-cracks ${ }^{5-6}$. By this process, the laser damage performance can be improved, which greatly depends on the process parameters. Most studies have shown that the laser-induced damage threshold (LIDT) of optical glass (e.g., fused silica, K9 glass) increases after slight etching, while it decreases after excess etching ${ }^{7-8}$. The possible reasons for this have been hypothesized by most researchers. The most recognized reasons or hypotheses are that (1) the absorptive contaminants (e.g., Ce, etc) located in polishing layer can be removed by slight etching, which results in the increase of LIDT $^{9}$; (2) some SSDs below the polishing layer are exposed and reaction products (RPs) redeposit after excess etching, which leads to the decrease of LIDT $^{10}$. Reason (1) has been validated experimentally by most researchers. They found that absorptive contaminants, especially some metallic ions, would lead to a localized optical absorption below the bandgap of optical glass ultimately causing laser damage under the irradiation of high-power laser. However, reason (2) is just a hypothesis. Although some researchers have proved that RPs could cause the laser damage, they usually considered RPs as metal salts (e.g., Fe, Al, etc) ${ }^{11-12}$. In fact, the absorption coefficient of these metal salts is not very high compared with that of $\mathrm{Ce}$ ion, which has a very weak influence on laser damage performance ${ }^{9}$. In addition, new research has shown that the LIDT may be improved if SSDs are etched excessively ${ }^{13}$. Therefore, it is necessary to investigate the effects of micro-crack and RP on laser damage performance again and reveal their effect mechanism. 
In this paper, four K9 glasses are prepared and etched. During their chemical etching, the morphologies of cracks and RPs are characterized, and the LIDTs are measured. Using finite-difference time-domain (FDTD) method, the light intensities around crack, RP and their combination ("crack + RP", C-RP for short) are simulated under different geometric parameters, respectively. Both experiments and simulations reveal the effect mechanism of crack and RP on the LIDT. This work could be beneficial to optimizing chemical etching process to improve the LIDT of optical glass.

\section{EXPERIMENT}

\subsection{Preparation of samples}

Two K9 glass blanks and two polished $\mathrm{K} 9$ glasses are prepared $\left(\mathrm{K} 9: \mathrm{SiO}_{2}=69.13 \%, \mathrm{~B}_{2} \mathrm{O}_{3}=10.75 \%, \mathrm{BaO}=3.07 \%\right.$, $\mathrm{Na}_{2} \mathrm{O}=10.40 \%, \mathrm{~K}_{2} \mathrm{O}=6.29 \%, \mathrm{As}_{2} \mathrm{O}_{3}=0.36 \%$ ), the roughnesses of which are about $20 \mathrm{~nm}$ and $1 \mathrm{~nm}$ respectively. These glasses are numbered (glasses 1, 2, 3 and 4). The glass blanks (glasses 1 and 2) are ground firstly by CNC Machining Center (S6150A, LOKSHUN), and then lapped with silicon carbide abrasive $(\sim 20 \mu \mathrm{m}$ of median size $)$ and polished with rare earth polishing powder (TREO, 98; $\mathrm{CeO}_{2}, 94 ; \mathrm{F}, \geq 5.0 ; \mathrm{pH}, 6.5-7.5 ; \mathrm{D} 50,1.0-1.2 \mu \mathrm{m} ; \mathrm{D} 100: 2.9 \mu \mathrm{m}$ ) using the 9S glass grinder (YM-380SM). After these processes, cracks are generated in the glass with a size of $\Phi 57 \times 4 \mathrm{~mm}$. Two polished glasses (glasses 3 and 4) with a size of $\Phi 25.4 \times 4 \mathrm{~mm}$ are customized, which have no micro-cracks.

\subsection{Etching and measurements}

Each glass is etched five times with the solutions of $\mathrm{HF} / \mathrm{HNO}_{3}(80 / 20 \% \mathrm{v}$.), and each etching time is $0.5 \mathrm{~min}, 1.5 \mathrm{~min}, 2.5$ min, 3.5 min and 4.5 min, respectively. Ce ion and other contaminants can be reduced absolutely with strong acid $\mathrm{HNO}_{3}$. Glasses 1 and 3 are etched statically, and glasses 2 and 4 are etched dynamically with high-frequency ultrasonic agitation. RPs would redeposit on the glass surface after static etching, while they are mitigated significantly during the dynamic etching. The surface morphology and laser damage performance of each glass are measured after each etching experiment, respectively. Meanwhile, before each measurement, each glass is cleaned with alcohol, acetone, and deionized water, respectively, for $5 \mathrm{~min}$ in an ultrasonic cleaning machine.

The laser scanning confocal microscopy (OLS4000, Olympus Corporation, Japan) is used to observe the surface morphology. The damage performance is tested by LIDT test platform, as shown in Fig. 1. A Nd:YAG laser operates at a wavelength of $1064 \mathrm{~nm}$ with pulse duration $10 \mathrm{~ns}$. The spatial beam profile is Gaussian shape with a $1 / \mathrm{e}^{2}$ diameter of 0.8 $\mathrm{mm}$. The laser energy attenuator consists of a rotatable half-wave and a polarization plate. After the light from Nd:YAG laser passes through the attenuator, a portion of light is reflected and directed to the energy detector, and another portion of light is focused on a sample by lens. The He-Ne laser outputs a $632.8 \mathrm{~nm}$ light, which would be scattered when it goes onto a damaged sample. Therefore, the damage can be detected by monitoring the energy change of scattered light. However, for simplicity, the damage is detected by CCD camera in this paper. When there is a change of 5 pixels before and after laser irradiation, the damage is defined as having occurred. According to standard ISO 11254-1, 1-on-1 test procedure is utilized to test the LIDT.

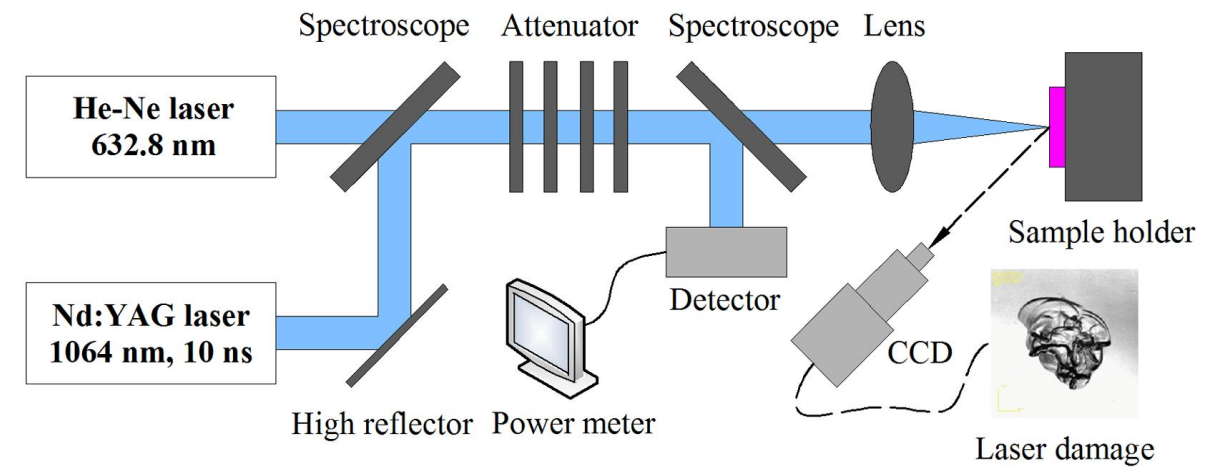

Figure 1. Schematic of LIDT test platform. 


\section{EXPERIMENTAL RESULTS AND DISCUSSIONS}

\subsection{Characterization of crack and RP}

Figure 2(a) shows the surface morphology of glass 1 before etching. Obviously, the initial glass surface is very smooth and surface fractures cannot be observed. This may be because the crack layer is hidden under the polishing layer. Figures 2(b)-2(f) show a randomly selected series of microscope images of glass 1 after etching for different times. It can be found that some cracks are exposed and distributed on the glass surface after chemical etching. It can be also found that the crack gradually becomes from a slender pit to a round pit with the increase of etching time. Figure 2(f) shows that there are many RPs on the glass surface, especially in the etched cusps. This phenomenon is consistent with the former research by Suratwala ${ }^{10}$, who found that RPs deposited in the etched cusps after static etching. He also found that RPs were reduced dramatically with the addition of agitation. Figure $2(\mathrm{~g})$ shows the microscope image of glass 2 after dynamic etching for $12.5 \mathrm{~min}$, indicating that there is almost no RP on the glass surface. Therefore, we assume that (1) there are many cracks and RPs on the surface of glass 1; (2) there are many cracks but no RP on the surface of glass 2; (3) there are many RPs but no crack on the surface of glass 3; and (4) there is no RP or crack on the surface of glass 4 during the whole chemical etching.

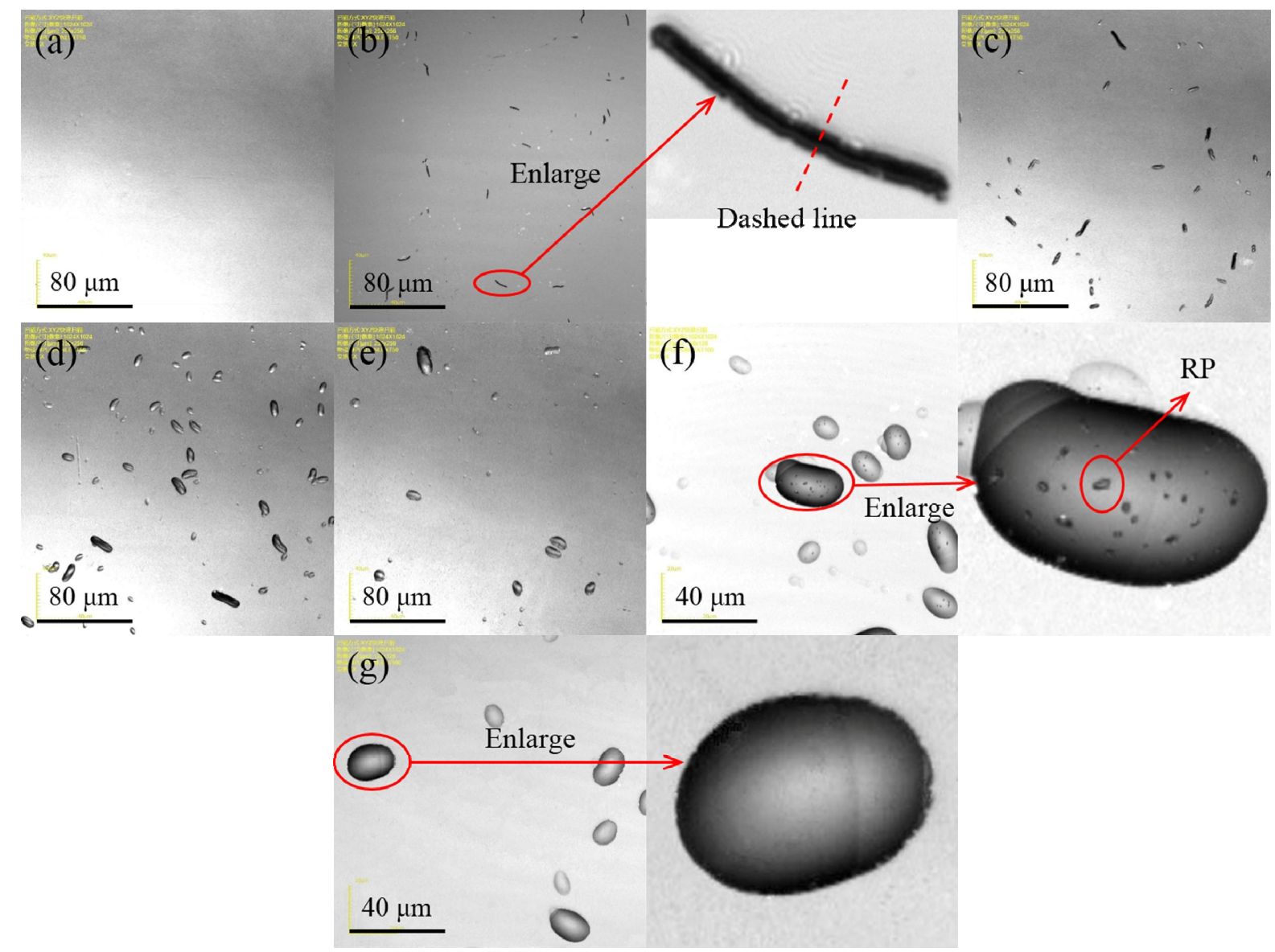

Figure 2. Morphologies of glass 1 after etching for (a) 0 min (initial surface); (b) $0.5 \mathrm{~min}$; (c) $2 \mathrm{~min}$; (d) $4.5 \mathrm{~min}$; (e) 8 min; (f) $12.5 \mathrm{~min}$. (g) Morphology of glass 2 after etching for $12.5 \mathrm{~min}$.

\subsection{Laser-induced damage threshold}

Figure 3 shows the LIDTs of four glasses after etching for different times. It can be found that the LIDTs of glasses 3 and 4 are larger than those of glasses 1 and 2 when the etching time is 0 min. It can be also found that after etching for $0.5 \mathrm{~min}$, the LIDTs of four glasses increase obviously whether it is a static etching or dynamic etching. This indicates 
that the LIDT can be improved after slight etching. However, as the chemical etching continues, the LIDT begins to decrease gradually for the glass with RPs (i.e., glasses 1 and 3), and the LIDT of glass 1 reduces more than that of glass 3 under the same etching time. In addition, the LIDT increases slowly and then stabilizes for the glass without RPs (i.e., glasses 2 and 4) as the chemical etching progresses. Figure 3 also shows the damage morpology after laser irradation.

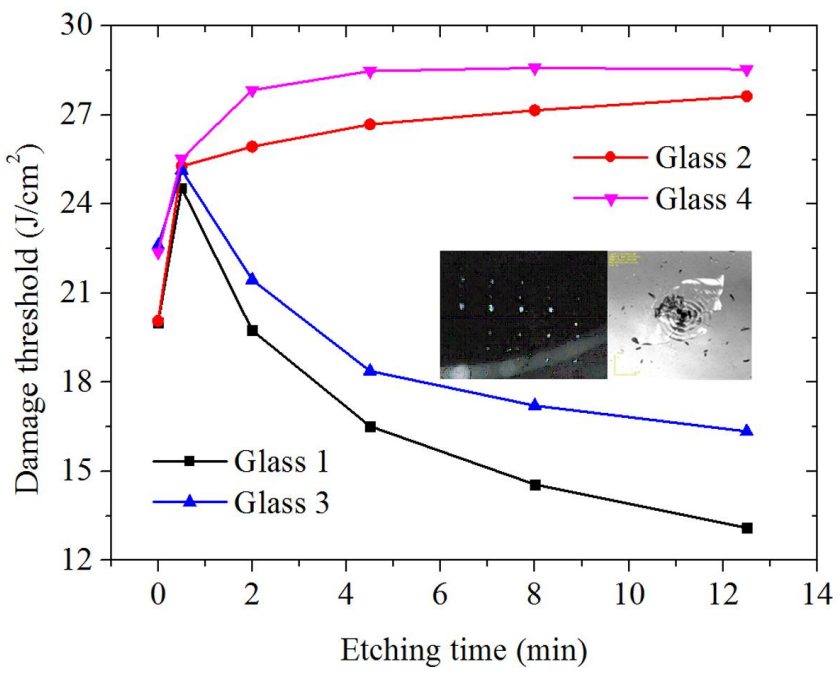

Figure 3. LIDTs of four glasses after different etching times.

\subsection{Effect mechanism of crack and RP}

Numerical simulation is a good method to theoretically investigate the influence of different kinds of defects on the laser damage performance by analyzing the light intensity around the defects. The light intensity is proportional to the square of electric field $\left(|\mathbf{E}|^{2}\right)$, which can be evaluated by LIEF. LIEF is defined as the ratio of the maximum light intensity caused by defects to the light intensity inside the smooth glass. In this paper, two-dimensional (2D) FDTD method is employed to solve the Maxwell's equations to obtain the light intensity around crack, RP and C-RP. Its aim is to reveal their light-field modulation mechanism. The simulation domain is a rectangular and gridded with a uniform grid $\delta=$ $\lambda / 12$, where the wavelength $\lambda$ equals to $1064 \mathrm{~nm}$. The relative dielectric constant (RDC) of glass is $2.25(\varepsilon=2.25)$. A plane wave with TE mode is used and its amplitude is normalized to $1 \mathrm{~V} / \mathrm{m}$. Since the laser damages on front and rear surfaces behave differently, the position of crack, RP and C-RP (on front or rear surface) is also considered.

According to the previous researches, a crack and a RP can be approximately regarded as a triangular and a circular geometries, respectively ${ }^{14-15}$. Here, we firstly define the geometric parameters of crack, RP and C-RP. Crack parameters include the spacing $(s)$, depth $(h)$ and width $(w)$ or aspect ratio $(g=h / w)$. RP parameters include the radius $(r)$, spacing $(s)$, embedded depth $(d)$, and RDC $(\varepsilon)$. C-RP parameter only includes the relative distance $(l)$ between the crack and the RP. All parameters are described in Fig. 4. Figure 4 also shows the distribution of relative light intensity around two rear-surface cracks, two rear-surface RPs, two front-surface RPs, and one rear-surface C-RP under the certain geometric parameters. Here, the relative light intensity is defined as the ratio between the light intensity induced by defects with that inside the smooth glass. Figure 4(a) shows that there are a series of standing waves inside the glass and a series of hot spots parallel to the rear surface, which is caused by the interference between the incident light and the reflected light from the cracks and glass surface. Figures 4(b) and 4(c) show that the incident light is focused since the RDC of round RP $(\varepsilon=5)$ is larger than that of glass. The light focusing may appear behind the RP (Fig. 4(b)) or inside the RP (Fig. 4(c)), which would introduce a light intensity enhancement domain. Because the RP plays a role in light focusing, it could be predicted that the LIEF for two RPs changes slightly with the RP spacing. Meanwhile, it is impossible for RP to embed into the glass because it is wet and soft during chemical etching. Therefore, it is not necessary to analyze the effect of RP spacing and embedded depth on the LIEF. Figure 4(d) shows an irregular light intensity distribution around one C-RP. 

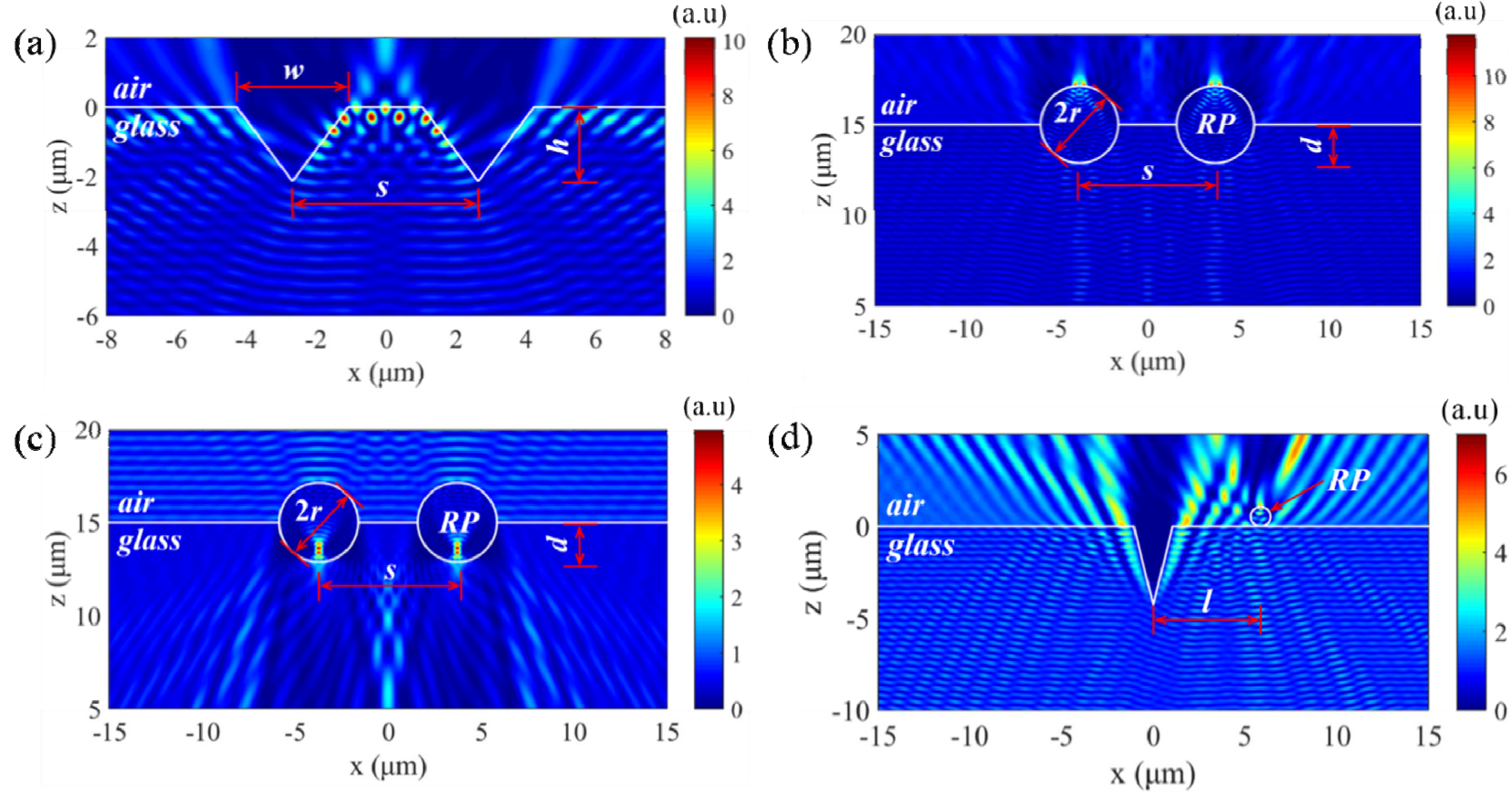

Figure 4. Distribution of relative light intensity around (a) two rear-surface cracks $(w=3 \lambda, h=2 \lambda, s=5 \lambda)$; (b) two rearsurface RPs; (c) two front-surface RPs $(r=2 \lambda, h=2 \lambda, s=7 \lambda, \varepsilon=5)$; and (d) one rear-surface C-RP $(w=2 \lambda, h=4 \lambda, r=$ $0.5 \lambda, \varepsilon=3, l=5.5 \lambda)$.

Figure 5 shows the LIEFs in all (i.e., in "air + glass") and in glass for two cracks under different crack spacings and aspect ratios. It can be found that the LIEF for cracks on rear surface is larger than that on front surface, and the LIEF in all is slightly larger than that in glass for rear-surface cracks. However, the LIEF in all have a small difference with that in glass for front-surface cracks. Here, we mainly focus on the LIEF in glass. Figures 5(c) and 5(d) reveal that the LIEF for two cracks is generally larger than that for one crack $(s=0)$ under the same aspect ratio. Figures $5(\mathrm{c})$ and $5(\mathrm{~d})$ also reveal that with the increase of crack spacing, the LIEF for two cracks generally increases firstly and then decreases when aspect ratio $g=2: 1$ to $2: 4$, while it generally increases at first, then declines and finally fluctuates around a small value when aspect ratio $g=2: 5$ to $2: 6$. It is easy to imagine that when the crack spacing tends toward infinity or zero, the LIEF for two cracks approximately equals to that for one crack. From this point, with the increase or decrease of crack spacing, the LIEF for two cracks generally decreases and ultimately fluctuates around the LIEF for one crack. Actually, this conclusion has been reached in our previous study, but in which we do not consider the LIEF for front-surface cracks $^{14}$. Observing the morphologies of glass after etching shown in Fig. 2, we find that the crack spacing is much far greater than the crack width, so it is not necessary to consider the effect of crack spacing on LIEF or LIDT in our experiments. Figure 5(d) shows that the LIEFs for one rear-surface crack are sorted from big to small: $g=2: 3, g=2: 2, g$ $=2: 4, g=2: 1, g=2: 5, g=2: 6$. Our previous study has shown that with the increase of etching time, the crack width increases while the crack depth remains unchanged, which would lead to a decrease of aspect ratio ${ }^{16}$. Therefore, for one crack with a large aspect ratio, which usually occurs in the actual crack, the LIEF would increase at first and then decrease as chemical etching progresses. This would result in a decrease at first and an increase of LIDT. It seems to contradict the experimental result that LIDTs increase after slight etching, as shown in Fig. 3. But, in fact, it indirectly points out that the main reason for the increase of LIDT (all four glasses) is the elimination of absorptive contaminants in polishing layer but not the mitigation of cracks after slight etching. Using the laser scanning confocal microscopy, we can measure the crack depth and width along the dashed line shown in Fig. 2(b). They are $0.318 \mu \mathrm{m}$ and $1.026 \mu \mathrm{m}$ respectively, so the aspect ratio $g=0.31<2: 6$. This means that the LIEF would be a very small value, and we can neglect the effect of crack on the LIEF or LIDT after etching for $0.5 \mathrm{~min}$. This indirectly indicates that the main reason for the further increase of LIDT (glasses 2 and 4) may be the further dissolution of other contaminants. 
(a)
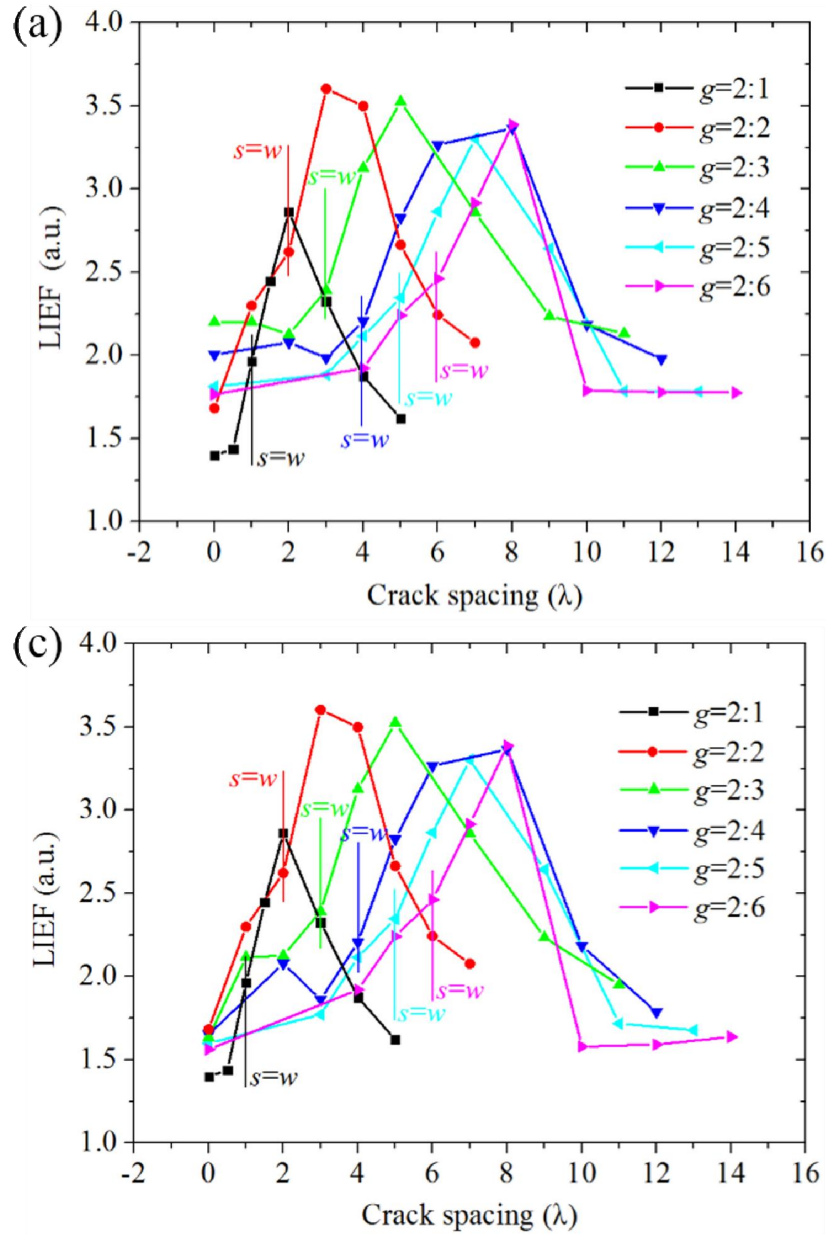
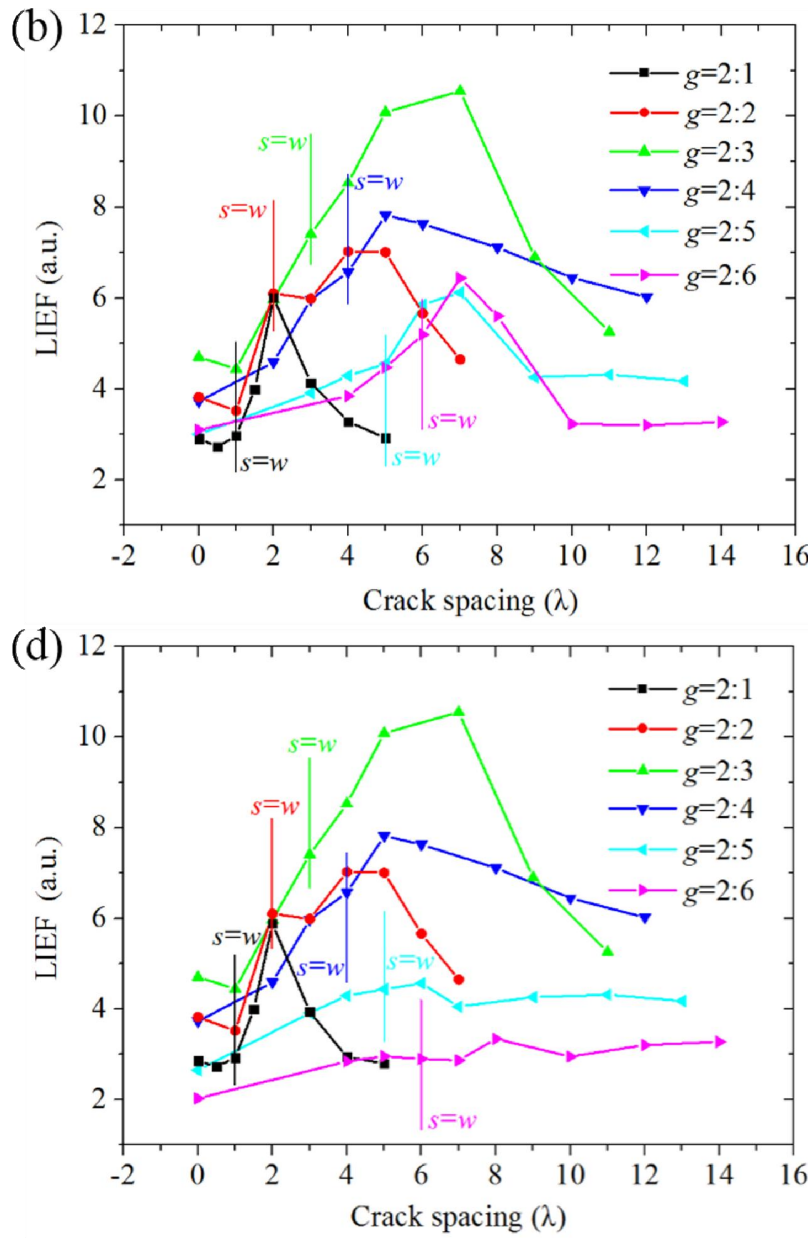

Figure 5. LIEFs in all for two cracks on (a) front surface and (b) rear surface; LIEFs in glass for two cracks on (c) front surface and (d) rear surface. LIEFs are calculated under different crack spacings and aspect ratios.

Figure 6 shows the LIEFs in all (i.e., in "air + glass + RP") and in glass for one RP under different RP radiuses and RDCs. It reveals that the LIEF increases with RP radius, and the LIEF in all is larger than that in glass under the same RP radius. Figure 6(a) displays that the LIEF in all fluctuates while the LIEF in glass decreases obviously with the increase of RDC for one front-surface RP. Figure 6(b) indicates that the LIEF in all or in glass fluctuates with the RDC for one rear-surface RP. Comparing Figs. 6(a) and 6(b), we can find that (1) the LIEF in all for one RP on rear surface is larger than that on front surface under the same RP radius; (2) the LIEFs in glass for one RP on front surface are larger and smaller than those on rear surface when RDC is relatively small and large, respectively. The above analyses can be utilized to explain the decrease of LIDT (glasses 1 and 3) with the increase of etching time. On the one hand, as chemical etching progresses, the RP deposition amount becomes more and more, the RP radius becomes larger and larger, resulting in an increase of LIEF and a decrease of LIDT. On the other hand, as shown in Fig. 2, more RPs would deposit in the etched cusps, making that the LIDT of glass 1 reduces more than that of glass 3 under the same etching time. In addition, the RDP of RP is usually closed to that of glass (i.e., 2.25). In this situation, the LIEF in glass for front-surface $\mathrm{RP}$ is relatively large, which would introduce a small LIDT.

Figure 7 shows the LIEFs in all (i.e., in "air + glass + RP") and in glass for one C-RP under different relative distances. Other geometric parameters have been determined $(w=2 \lambda, h=4 \lambda, r=0.5 \lambda$, and $\varepsilon=3)$. There are 62 distances ranging from 0 to $9 \lambda$, and the LIEF is simulated at each distance. From Fig. 7, we can find that the LIEF in all is generally larger than that in glass for one C-RP. When $l>w / 2$, the LIEF for one C-RP fluctuates with the distance. The fluctuation amplitude generally decreases with the increase of distance for one rear-surface C-RP. When $l=w / 2$, the LIEF for one $\mathrm{C}-\mathrm{RP}$ in glass has a peak. This may be utilized to explain the experimental result by Suratwala ${ }^{10}$. He found that the laser 
damage almost always initiated at the edge of the etched cusps, where RPs were more likely to deposit. We mainly focus on the LIEFs in glass. Figure 7 shows that the LIEFs in glass for one front-surface crack and RP are 2.95 and 3.50 respectively, and the LIEFs in glass for one rear-surface crack and RP are 4.84 and 2.64, respectively. Obviously, the larger values are 3.50 and 4.84 for front and rear surfaces, respectively. Statistics show that there are 39 distances and 61 distances, at which the LIEFs in glass for one C-RP are larger than 3.50 and 4.84, respectively. It means that the LIEF in glass for one C-RP is larger than that for one crack or one RP in most situations, which greatly depends on the relative distance. Therefore, we need to consider the effects of crack and RP on the LIEF simultaneously.
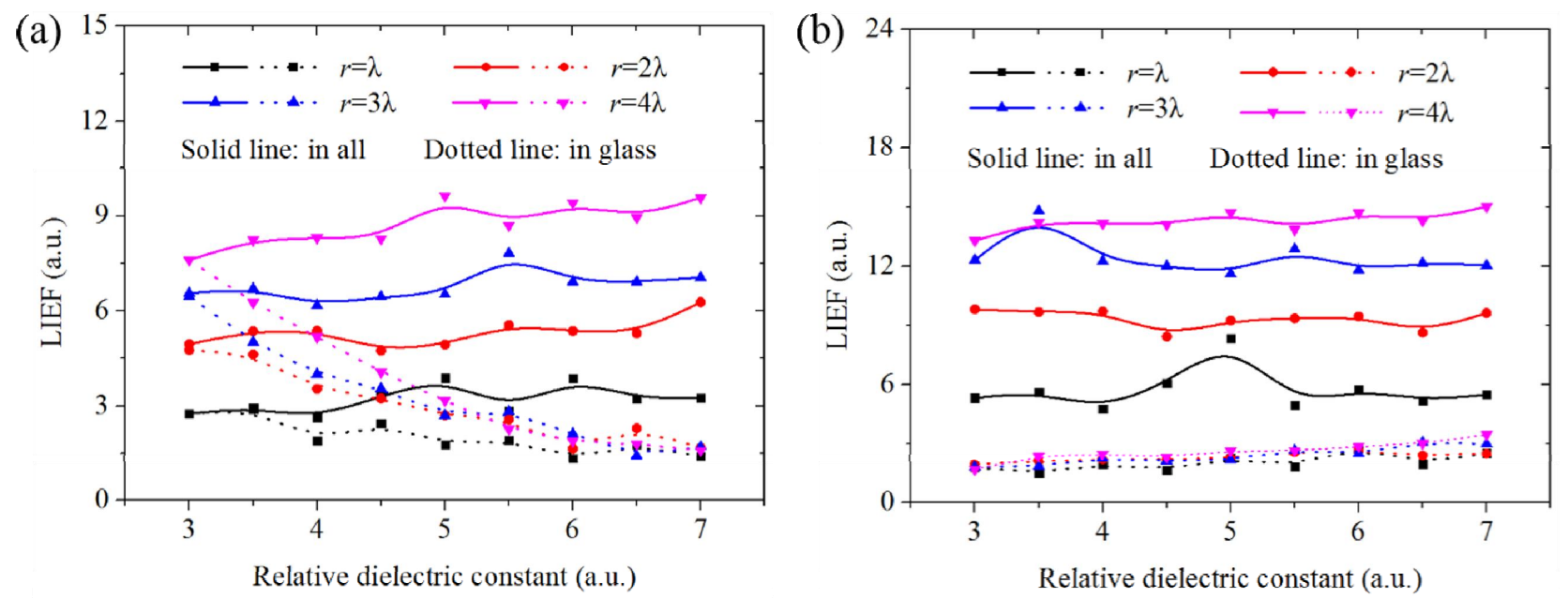

Figure 6. LIEFs in all and in glass for one RP on (a) front surface and (b) rear surface under different RP radiuses and RDCs.
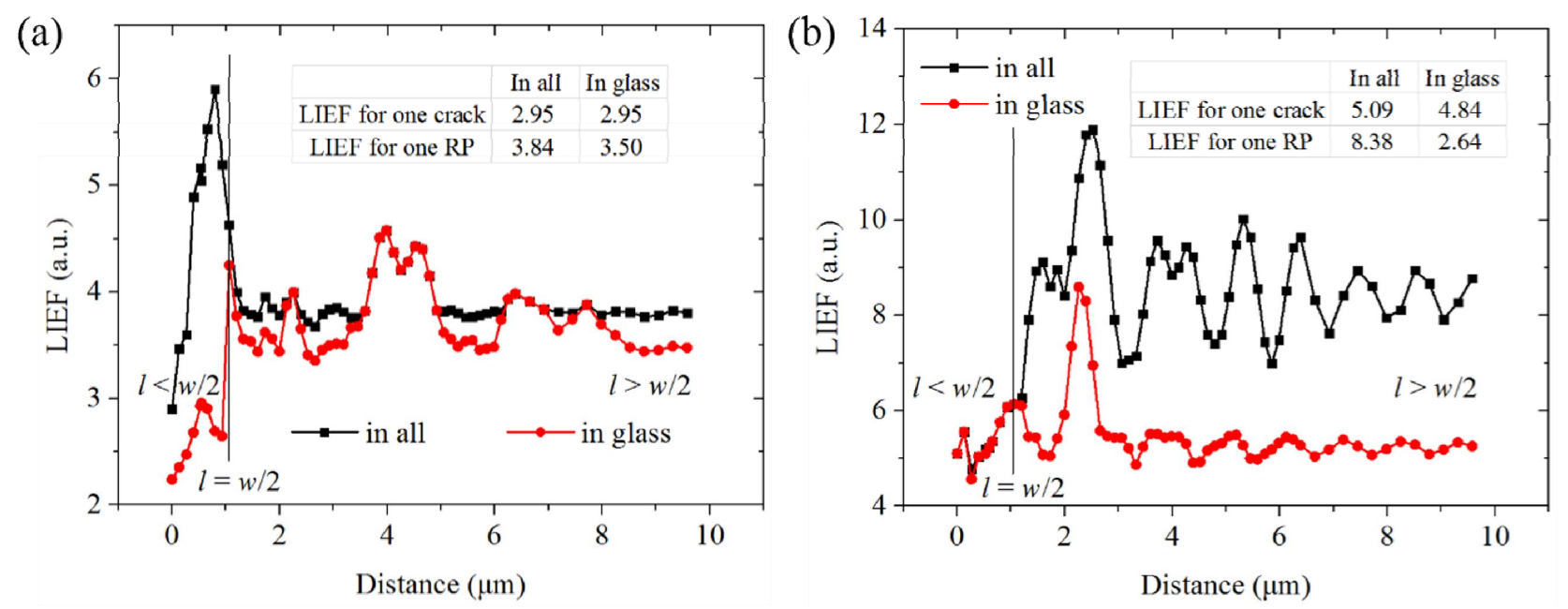

Figure 7. LIEFs in all and in glass for one C-RP on (a) front surface and (b) rear surface under different relative distances. Other geometric parameters have been determined, $w=2 \lambda, h=4 \lambda, r=0.5 \lambda$, and $\varepsilon=3$.

\section{CONCLUSION}

This paper has investigated the effect of crack and RP on the laser damage performance of K9 glass during chemical etching by experiments and simulations. Experiments show that the LIDT increases greatly after slight etching. However, as the chemical etching continues, the LIDT begins to decrease for the glass with RPs, and LIDT increases slowly and then stabilizes for the glass without RPs. Simulations show that the LIEF for one rear-surface crack with a high aspect ratio increases at first and then decreases with the increase of etching time, indirectly indicating that the main reason for the increase of LIDT is the elimination of contaminants but not the mitigation of cracks. Simulations also show that the LIEF increases with RP radius, and the LIEF is relatively large when the RDP of RP is close to that of glass. This 
explains why the LIDT decreases for the glass with RPs. In addition, simulation results indicate that the LIEF in glass for one C-RP is larger than that for one crack or one RP in most situations. This work could be utilized as guidelines to optimize optical fabrication and improve the laser damage performance of optical component.

\section{ACKNOWLEDGMENT}

This work is supported by The National Natural Science Foundation of China (Grants No. 51175416 and No. 51675420), and The National Key Research \& Development (R\&D) Program of China (Grant No. 2016YFB0501604-02).

\section{REFERENCES}

[1] S. Y. Li, Z. Wang, and Y. L. Wu, "Relationship between subsurface damage and surface roughness of optical materials in grinding and lapping processes," J. Mater. Process. Technol. 205, 34-41 (2008).

[2] H. R. Wang, H. F. Chen, G. L. Fu, and H. P. Xiao, "Relationship between grinding process and the parameters of subsurface damage based on the image processing," Int. J. Adv. Des. Manuf. Technol. 83, 1707-1715 (2016).

[3] Y. G. Li, H. Ye, Z. G. Yuan, Z. C. Liu, Y. Zheng, Z. Zhang, S. J. Zhao, J. Wang, and Q. Xu, "Generation of scratches and their effects on laser damage performance of silica glass," Sci. Rep. 6, 34818 (2016).

[4] M. L. Lu, B. Ma, G. D. Zhan, H. F. Jiao, and X. B. Cheng, "Effect of etching on the laser-induced damage properties of artificial defects under 1064-nm laser irradiation," Opt. Eng. 53(12), 122505 (2014).

[5] M. Kolli, M. Hamidouche, N. Bouaouadja, and G. Fantozzi, "HF etching effect on sandblasted soda-lime glass properties," J. Eur. Ceram. Soc. 29, 2697-2704 (2009).

[6] F. M. Ezz-Eldin, T. D. Abd-Elaziz, and N. A. Elalaily, "Effect of dilute HF solutions on chemical, optical, and mechanical properties of soda-lime-silica glass," J. Mater. Sci. 45, 5937-5949 (2010).

[7] Z. Zheng, X. T. Zu, X. D. Jiang, X. Xiang, J. Huang, X. D. Zhou, C. H. Li, W. G. Zheng, and L. Li, "Effect of HF etching on the surface quality and laser-induced damage of fused silica," Opt. Laser. Technol. 44, 10391042 (2012).

[8] H. Ye, Y. G. Li, Z. G. Yuan, J. Wang, Q. Xu, and W. Yang, "Improving UV laser damage threshold of fused silica optics by wet chemical etching technique," Proc. SPIE 9532, 1-6 (2015).

[9] X. Ye, J. Huang, H. J. Liu, F. Geng, L. X. Sun, X. D. Jiang, W. D. Wu, L. Qiao, X. T. Zu, and W. G. Zheng, "Advanced mitigation process (AMP) for improving laser damage threshold of fused silica optics," Sci. Rep. 6, 31111 (2016).

[10] T. I. Suratwala, P. E. Miller, J. D. Bude, W. A. Steele, N. Shen, M. V. Monticelli, M. D. Feit, T. A. Laurence, M. A. Norton, C. W. Carr, and L. L. Wong, "HF-based etching processes for improving laser damage resistance of fused silica optical surfaces," J. Am. Ceram. Soc. 94(2), 416-428 (2011).

[11]H. Ye, Y. G. Li, Q. H. Zhang, W. Wang, Z. G. Yuan, J. Wang, and Q. Xu, "Post-processing of fused silica and its effects on damage resistance to nanosecond pulsed UV lasers," Appl. Opt. 55(11), 3017-3025 (2016).

[12]Z. Q. Wang, H. W. Yan, X. D. Yuan, K. Yang, Y. Li, L. H. Yan, L. J. Zhang, T. X. Liu, and H. Y. Li, "Formation of redeposit in chemical etching process of fused silica and its effect on laser-induced damage," High Power Laser and Particle Beams 29(4), 041001 (2017). (in Chinese)

[13] J. Huang, X. D. Zhou, H. J. Liu, F. R. Wang, X. D. Jiang, W. D. Wu, Y. J. Tang and Y. G. Zheng, "Influence of subsurface defects on damage performance of fused silica in ultraviolet laser," Opt. Eng. 52(2), 024203 (2013).

[14]H. P. Xiao, H. R. Wang, Z. Chen, G. L. Fu, and J. H. Wang, "Effect of brittle scratches on transmission of optical glass and its induced light intensification during the chemical etching," Opt. Eng. 56(10), 105101 (2017).

[15] L. Yang, X. Xiang, X. X. Miao, L. Li, X. D. Yuan, Z. H. Yan, G. R. Zhou, H. B. Lv, W. G. Zheng, and X. T. Zu, "Numerical simulation of modulation to incident laser by submicron to micron surface contaminants on fused silica," Chin. Phys. B 25(1), 014210 (2016).

[16]H. P. Xiao, H. R. Wang, G. L. Fu, and Z. Chen, "Surface roughness and morphology evolution of optical glass with micro-cracks during chemical etching," Appl. Opt. 56(3), 702-711 (2017). 